\title{
Atomic structure and chemistry of a[100] dislocation cores in
}

\section{$\mathrm{La}_{2 / 3} \mathrm{Sr}_{1 / 3} \mathrm{MnO}_{3}$ films}

\author{
Kepeng Song, Kui Du*, Hengqiang Ye
}

Shenyang National Laboratory for Materials Science, Institute of Metal Research, Chinese Academy of Sciences, Shenyang 110016, China

\begin{abstract}
Oxide thin films with perovskite structures possess multifunctional properties, while defects in the films usually have significant influences on their physical properties. Here, the atomic structure and chemistry of a[100] dislocation cores in epitaxial $\mathrm{La}_{2 / 3} \mathrm{Sr}_{1 / 3} \mathrm{MnO}_{3}$ films were investigated by aberration-corrected scanning transmission electron microscopy combining with atomically resolved electron energy-loss spectroscopy imaging. The results demonstrated an edge dislocation terminated with Mn columns and significant nonstoichiometry at the dislocation core region. Quantitative analysis using core-loss spectrum indicates that $\mathrm{La} / \mathrm{Mn}$ and $\mathrm{O} / \mathrm{Mn}$ ratios are decreased at the dislocation core. Antisite defects with Mn ions at La-sites were directly determined at the dislocation cores with electron energy-loss spectroscopy. The structure of the dislocation core is discussed on the basis of high-angle annular dark-field imaging and electron energy loss spectroscopy results.
\end{abstract}

Keywords: $\mathrm{La}_{2 / 3} \mathrm{Sr}_{1 / 3} \mathrm{MnO}_{3}$, dislocation, STEM, EELS, antisite. 


\section{Introduction}

Materials with the $\mathrm{ABO}_{3}$ perovskite structure have been found to possess multifunctional properties such as superconductivity (Klie et al., 2005; Reyren, 2007), ferroelectricity (Cohen, 1992), ferromagnetism and anti-ferromagnetism (Lee et al. 2010), colossal magnetoresistance (CMR) (Millis et al. 1996) and so on. Two dimensional perovskite films often exhibit unique properties different from the parent bulk materials because of the reduced dimensionality. These unique properties are related with not only the interfacial effects but also influences from other defects, such as point defects, stacking faults and dislocations. Thus, knowledge on the atomic structure of thin films is important to understand their structure-property relationships and to optimize the film quality.

Structure of defects such as dislocations and point defects in functional materials is one of the main topics in materials research. Although dislocation cores are limited to rather small dimensions, they usually have significant influences on physical properties such as magnetic resistance, electronic and ionic transport of materials due to their special atomic and electronic structures, chemistry and strain states at the dislocation cores. For example, investigations on $\mathrm{SrTiO}_{3}$ (Kim, 2001; Zhang, 2002, 2004), $\mathrm{BaTiO}_{3}$ (Kurata, 2008) and $\mathrm{PbZr}_{0.52} \mathrm{Ti}_{0.48} \mathrm{O}_{3}$ (Arredondo, 2010) show that dislocation cores are regions with distinct chemistry from bulk regions. More recently, ferromagnetic dislocations in antiferromagnetic $\mathrm{NiO}$ have been discovered and this unexpected emergence of ferromagnetism at dislocations originates from local 
non-stoichiometry at the dislocation cores with Ni deficiency (Sugiyama, 2013). Thus, it is critical to determine the structure and chemistry of dislocation cores in oxide thin films at atomic resolution.

Scanning transmission electron microscopy (STEM), combined with electron energy-loss spectroscopy (EELS) provide the capability to directly investigate the local atomic structure and chemistry at the atomic scale with high spatial and energy resolutions (Pennycook, 1988; Varela, 2004; Bosman, 2007; Muller, 2009). Here, we report on direct atomic resolution imaging of the core structure of a[100] edge dislocations in $\mathrm{La}_{2 / 3} \mathrm{Sr}_{1 / 3} \mathrm{MnO}_{3}$ films deposited on $\mathrm{LaAlO}_{3}$ substrates by pulsed laser deposition through high angle annular dark field (HAADF) STEM and EELS spectra imaging with state-of-the-art aberration-corrected transmission electron microscope. The experimental results provide direct evidence of a dislocation core terminated with Mn columns and existence of antisite defects at the dislocation core.

\section{Material and methods}

Epitaxial $\mathrm{La}_{2 / 3} \mathrm{Sr}_{1 / 3} \mathrm{MnO}_{3}$ (LSMO) films (60 nm thick) were grown on 10 x 3 x $0.5 \mathrm{~mm}^{3}$ (001) orientated $\mathrm{LaAlO}_{3}$ (LAO) single crystal substrates by pulsed laser deposition (PLD). KrF excimer laser with wavelength of $248 \mathrm{~nm}$ was used. The energy density was $1.6 \mathrm{~J} / \mathrm{cm}^{2}$ and the repetition rate was $3 \mathrm{~Hz}$. During the crystal growth process, the substrate temperature was kept at $750{ }^{\circ} \mathrm{C}$ and the oxygen pressure was kept at 0.4 mbar. After the deposition, the films were annealed in oxygen pressure near one atmosphere at $600{ }^{\circ} \mathrm{C}$ for 30 minutes and then were cooled down to 
room temperature slowly. The nominal thickness was about $60 \mathrm{~nm}$.

Plan-view specimens for TEM observations were prepared by thinning from the substrate side, including slicing, mechanical polishing, dimpling and finally ion milling under liquid nitrogen temperature to decrease beam damage. Bright-field TEM images were taken at $200 \mathrm{kV}$ on a Tecnai F20 microscope (FEI Company, USA). Both HAADF-STEM images and STEM-EELS imaging were taken with a Titan-cubed G2 80-300 kV microscope (FEI Company, USA) equipped with double Cs-corrector and a GIF Quantum 965 system (Gatan Inc., USA) with dual-eels. The convergence angle was around $23 \mathrm{mrad}$, and HAADF STEM images were taken with 70-250 mrad scattering angle. Data analysis was performed with DigitalMicrograph software (Gatan Inc., USA).

\section{Results and discussion}

Threading dislocations run along the [001] direction may have a Burgers vector parallel to [100], [101] or [10-1] in perovskite thin films. Bright field TEM imaging has been conducted under a series of two-beam conditions. As shown in Figure 1(a-d), dislocations indicated by arrows are visible with $\mathbf{g}=(200),(1-11),(-24-2)$ and (24-2). According to the $\mathbf{g} \cdot \mathbf{b}$ criteria, it can be concluded that the threading dislocations are edge dislocations with the Burgers vector of [100]. Moreover, according to the generally believed relaxation mechanism of lattice misfit in heteroepitaxial perovskite thin films (Suzukia, 1999; Qin, 2002), the possibility for $45^{\circ}$ threading dislocations evolved in the [001] direction in plan-viewed specimens is very low, and pure edge 
$\mathbf{a}[100]$ and $\mathbf{a}[110]$ threading dislocations are commonly seen in heteroepitaxial perovskite thin films (Suzukia, 1999; Qin, 2002).
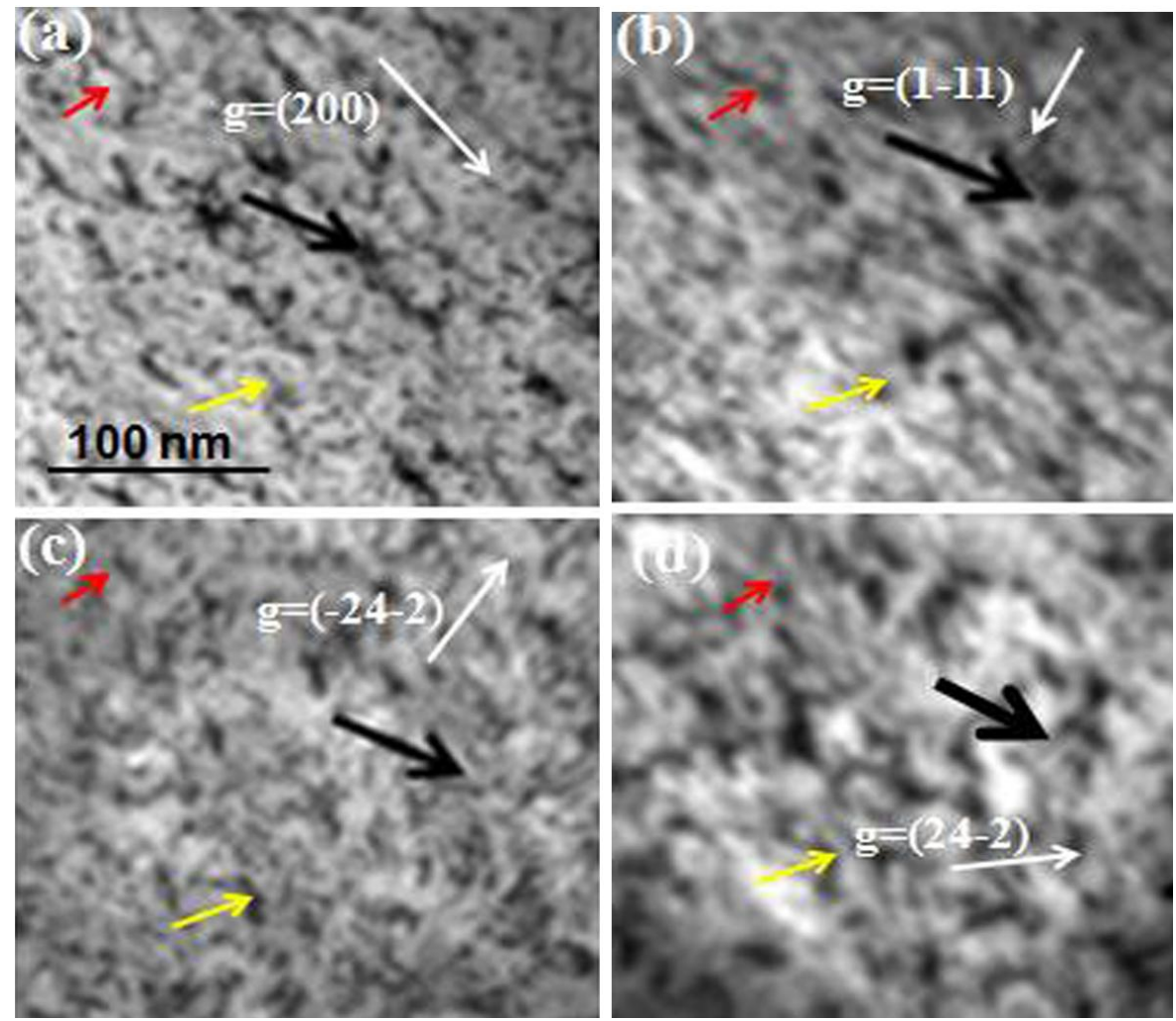

Figure 1. Plan-view bright field TEM micrographs under the conditions of $\mathbf{g}=(200)$

(a), $g=(1-11)(b), g=(-24-2)(c)$ and $g=(24-2)(d)$. Arrows indicate certain threading dislocations.

The lattice constants of LSMO and LAO are $0.387 \mathrm{~nm}$ and $0.379 \mathrm{~nm}$ respectively, and lattice mismatch between the thin film and the substrate is approximate $2.11 \%$ here. HAADF-STEM images can reveal atomic structure of materials and distinguish the types of atomic columns with significant difference in atomic numbers. Figure 2 presents a typical HAADF-STEM image of a threading dislocation in the LSMO film viewed along the [001] direction of a plane-view specimen. Burgers circuit was drawn around the dislocation core, where the failure of Burgers circuit around the dislocation core confirms that the threading dislocation is 
of the edge-type with a Burgers vector of $\mathbf{a}<100>$. In HAADF-STEM images, since the image contrast is approximately proportional to the square of the atomic number (Z) (Pennycook, 1988), stronger bright spots in the image correspond to $\mathrm{La}(\mathrm{Sr})$ column, whereas less bright ones are Mn-O colums. Nevertheless, the dislocation core region shows much darker contrast compared with the perfect matrix region. It has been reported that dislocation cores always show different contrasts in HAADF-STEM images, which might result from a relatively different chemistry composition, atom mixture, or a possible dechanneling effect from strain and orientation effects (Perovic, 1993; Chisholm, 2006). Thus, it is impossible to clearly distinguish the elements at the dislocation core directly from the HAADF-STEM image. Therefore, in order to investigate the chemistry of the dislocation core of LSMO (100) edge dislocations, atomic resolution STEM-EELS spectra imaging was performed over the dislocation core. 


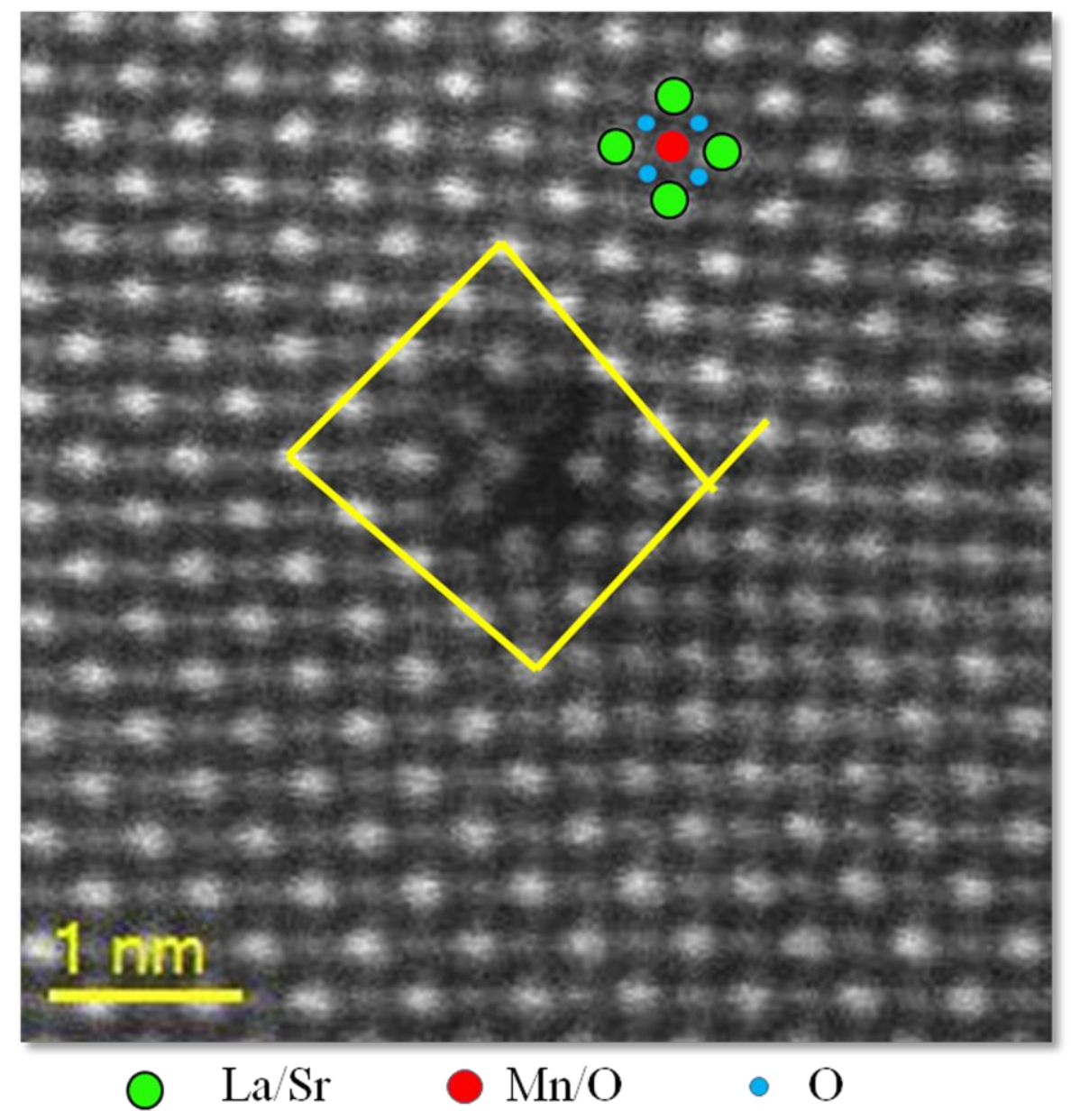

Figure. 2 HAADF-STEM image of a threading dislocation in LSMO thin film with the projected Burgers vector of $\mathbf{b}=[100]$ viewed along the [001] direction. LSMO perovskite structure model is superimposed on the HAADF-STEM image.

During the analysis of the spectra imaging, background subtraction for the elemental mapping extraction was carried out by fitting the pre-edge background with a power law $\mathrm{AE}^{-\mathrm{B}}$, where $\mathrm{A}$ and $\mathrm{B}$ are both fitting parameters (Egerton, 2011). Figure 3 shows the simultaneously acquired HAADF-STEM image and elemental maps. As a doping element, Sr usually lies in La columns and is difficult to map using its $\mathrm{M}$ edges due to the low energy and delayed onset, which makes it difficult to fit the 
pre-edge background. Besides, no significant Sr fluctuation was observed by EELS over the detecting regions. Therefore, $\mathrm{Sr}$ doping is not considered here. With Hatree-Slater model here, the $\mathrm{La} / \mathrm{Mn}$ ratio at the dislocation core was about $0.45 \pm 0.01$, which is much lower than the nominal ratio (0.67) in LSMO. More interestingly, from the combined La and Mn maps (Figure 3e), it is indicated that two Mn columns occupied the sites of La-sublattice in LSMO structures, where the two columns at the very dislocation core are Mn columns.

To quantitatively characterize the chemical compositions such as $\mathrm{La} / \mathrm{Mn}$ and $\mathrm{O} / \mathrm{Mn}$ atomic ratios, the Hatree-Slater model was used to calculate the cross section (Rez, 1982; Zhang, 2004). The O/Mn atomic ratio at LSMO matrix and dislocation core are determined as those displayed in Figure 3(f). The O/Mn ratio at the LSMO matrix (positions 1 and 3 in Figure 3(a)) is around 3 ( $3.17 \pm 0.22$ and $3.07 \pm 0.18$ at position 1 and 3 respectively), which is close to the nominal $\mathrm{O} / \mathrm{Mn}$ ratio $(\mathrm{O} / \mathrm{Mn}=3)$ in LSMO. This value demonstrates the accuracy of the above used Hatree-Slater model for the calculation of cross-section and also illustrates that the specimen may not suffer considerable electron beam damage during the STEM-EELS experiments. However, the $\mathrm{O} / \mathrm{Mn}$ ratio at the dislocation core is $2.72 \pm 0.23$, which is lower than that at LSMO matrix. Similarly, the $\mathrm{La} / \mathrm{Mn}$ ratio is $0.45 \pm 0.01$ at the dislocation core, which is significantly low compared with that in LSMO (nominal $\mathrm{La} / \mathrm{Mn}=0.67$ ). Based on these results, it is demonstrated that both $\mathrm{O} / \mathrm{Mn}$ and $\mathrm{La} / \mathrm{Mn}$ ratios are decreased at the dislocation core, but it is difficult to say whether $\mathrm{O}$ and $\mathrm{La}$ are deficient of $\mathrm{Mn}$ is in excess. The contrast of HAADF-STEM image in Fig. 1 might 
suggest a deficiency of heavy La atoms. In order to further determine the chemical compositions, the ELNES of O-K and $\mathrm{Mn}-\mathrm{L}_{2,3}$ are examined.
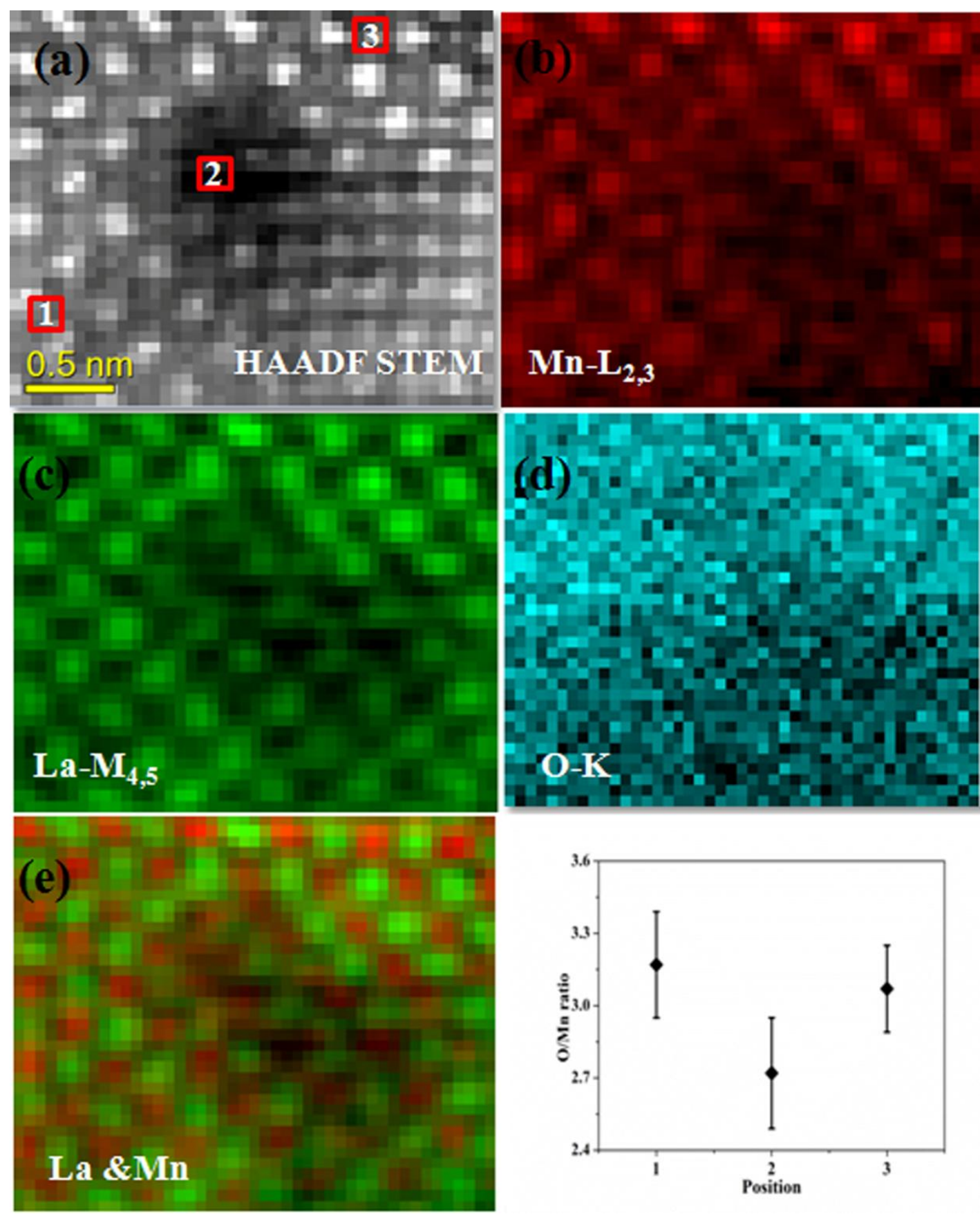

Figure. 3 Atomic-resolution EELS maps of the dislocation core, (a) the

simultaneous recorded HAADF-STEM image, (b) La-M $\mathrm{M}_{4,5}$ edges, (c) Mn- $\mathrm{L}_{2,3}$ edges, (d) O-K edge, (e) color-mixed mapping of La and Mn and (f) calculated O/Mn ratio 
from the 3 positions in (a), which show decrease at the dislocation core compared with the LSMO matrix.

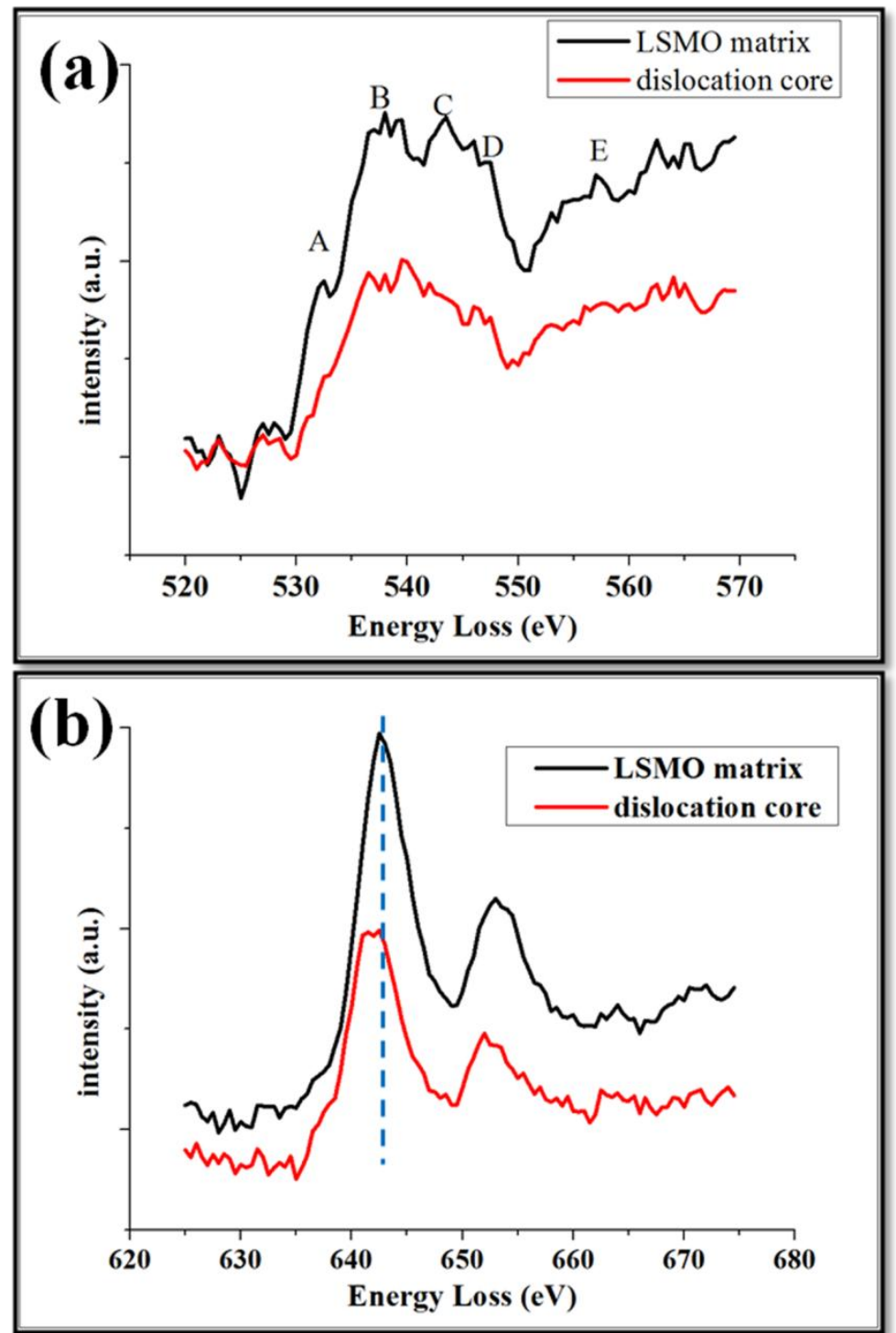

Figure. 4 Energy-loss near-edge structures (ELNES) of O-K edge (a) and $\mathrm{Mn}^{-\mathrm{L}_{2,3}}$ edges (b) extracted from the LSMO matrix (black) and the dislocation core (red).

L edges of transition metals reflect the empty local density of states of the $3 \mathrm{~d}$ orbitals and can be used to determine the valence states of transition metal ions. 
Quantitative analysis of the intensity ratio and the chemical shift can reveal the valence changes of transition metals (Schmid, 2006; Varela, 2009). Meanwhile, information on oxygen vacancy can be derived from the energy-loss near-edge structures (ELNES). Here, ELNES were extracted from the recorded spectra image. With the atomic resolution spectra image, EEL spectra can be precisely extracted from the dislocation core and LSMO matrix. O-K edge and $\mathrm{Mn}-\mathrm{L}_{2,3}$ edges were extracted and presented in Figure 4 (a) and (b) respectively. The fine structure of $\mathrm{O}-\mathrm{K}$ edge reflects information on excitations from $\mathrm{O} 1 \mathrm{~s}$ electron to $2 \mathrm{p}$ bands. There are three main peaks for LSMO: a pre-peak at about $530 \mathrm{eV}$ (labeled A), the first main peak at around $535 \mathrm{eV}(\mathrm{B})$ and a second main peak at about $540 \mathrm{eV}(\mathrm{C})$. The pre-peak is associated with the filling states of Mn $3 \mathrm{~d}$ states and is sensitive to Mn $3 \mathrm{~d} \mathrm{e}_{\mathrm{g}}$ state occupancies, while the first main peak reflects hybridization with $\mathrm{La} 5 \mathrm{~d}$ and $\mathrm{Sr} 3 \mathrm{~d}$ bands and the second peak provide information of the Mn 4sp bands. Comparing the O-K edge (Figure 4(a)) from LSMO matrix and the dislocation core, the intensity of the pre-peak can be found to decrease at the dislocation core, which indicated a reduction of Mn valence. Furthermore, the broadening of the first main peak suggest a decrease of La-O bonding due to La deficiency at the dislocation core. $\mathrm{Mn} \mathrm{L}_{2,3}$ edges represents electron transition from $\mathrm{Mn} 2 \mathrm{p}_{1 / 2}$ and $2 \mathrm{p}_{3 / 2}$ to unfilled $3 \mathrm{~d}$ states, and it has been demonstrated that the Mn valences are related with the $\mathrm{L}_{3} / \mathrm{L}_{2}$ ratio and this ratio decrease monotonically when the Mn valences change from 2+ to 7+ (Leapman, 1982; Varela, 2009). Here, with the Hatree-Slater cross section function as a step function to remove the continuum contribution, the $\mathrm{L}_{3} / \mathrm{L}_{2}$ ratios at $\mathrm{LSMO}$ matrix and the 
dislocation core are 2.34 and 2.47 respectively, which indeed shows an increase of the $\mathrm{L}_{3} / \mathrm{L}_{2}$ ratio at the dislocation core. Chemical shifts of $\mathrm{L}_{3}$ and $\mathrm{L}_{2}$ edges are also characteristics for the variation of Mn valences. Here, in Figure 4(b), it can be found that $\mathrm{L}_{3}$ and $\mathrm{L}_{2}$ peaks display a shift of approximately $0.8 \mathrm{eV}$ to the lower energy side. Both the $\mathrm{L}_{3} / \mathrm{L}_{2}$ intensity ratio variation and the chemical shift of $\mathrm{L}_{3}$ and $\mathrm{L}_{2}$ peaks here illustrate the reduction of Mn valence, which is consistent with the results deduced from the $\mathrm{O} \mathrm{K}$ edge.

Combining HAADF-STEM and atomic resolution EELS results, it is quite helpful to elucidate the structure of the dislocation core. The structure and chemistry of edge dislocations in the perovskite lattices, especially dislocation cores in $\mathrm{SrTiO}_{3}$ and $\mathrm{BaTiO}_{3}$ (Kim, 2001; Zhang, 2002, 2004; Kurata, 2008), have been extensively investigated, which showed that edge dislocations in these compounds are usually terminated with Ti-O columns and of oxygen deficiency. The atomic configuration of dislocation cores in LSMO here is not consistent with structures reported in $\mathrm{SrTiO}_{3}$ and $\mathrm{BaTiO}_{3}$, in which the edge dislocations were terminated with $\mathrm{Ti} / \mathrm{O}$ columns and the extra atomic planes were Ti/O planes. However, according to the above analysis, present results of atomic resolution STEM-EELS support the viewpoints that edge dislocation cores in LSMO films here are terminated with Mn columns and the extra atomic plane here are $\mathrm{La} / \mathrm{Sr}$ colums with two Mn colums at the dislocation core region. Figure 5 shows an enlarged HAADF STEM image around the dislocation core, which was superimposed with a structure model deduced from the atomic resolution STEM-EELS results. Oxygen columns are not displayed here because of their low 
scattering capabilities and it is difficult to jude the position and vacancy ratio at $\mathrm{O}$ columns. The dislocation is terminated with Mn columns at a La-O plane and only the two columns in the centre of the dislocation core (in circle) are proved to be $\mathrm{Mn}$ columns but occupy the La-sites in La-sublattice, that is to say that the dislocation cores are terminated with $\mathrm{Mn}_{\mathrm{La}}$ ( $\mathrm{Mn}$ in La site) antisite defects. Another interesting fact is that two sites indicated by white arrows in Figure 5 showed no contrast, which means there should be no cation-occupation. Considering the charge neutrality, this cation deficiency can partially explain the decreased La/Mn ratio, the valence reduction of $\mathrm{Mn}$ ions as well as the oxygen vacencies at dislocation core regions, as indicated by the quantitative analysis of the $\mathrm{O}-\mathrm{K}$ edge and $\mathrm{Mn} \mathrm{L}_{2,3}$-edges. Strain states at the dislocation core may also cause the nonstoichiometry as well. As is known, in LSMO perovskite structure, Mn ions with small radius occupy the B-site, which are the centre of an oxygen octahedron. The ionic radius of $\mathrm{La}^{3+}(0.103 \mathrm{~nm})$ is rather lager than that of Mn ions $\left(\mathrm{Mn}^{2+}: 0.067 \mathrm{~nm} ; \mathrm{Mn}^{3+}: 0.058 \mathrm{~nm} ; \mathrm{Mn}^{4+}: 0.053 \mathrm{~nm}\right)$. In order to accommodate the lattice distortion caused by the dislocation, Mn-O octahedra may collapse at the dislocation core, and consequently cause the empty columns (indicated by the white arrows in Fig. 4) as well as oxygen deficiency. Meanwhile, Mn ions can move into the La-site, which could further accommodate the lattice distortion. With the collapse of $\mathrm{Mn}-\mathrm{O}$ octahedra and the formation of $\mathrm{Mn}_{\mathrm{La}}$ antisite defects, it is thus possible to explain the nonstoichiometry and valence changes of $\mathrm{Mn}$ ions at the dislocations. 


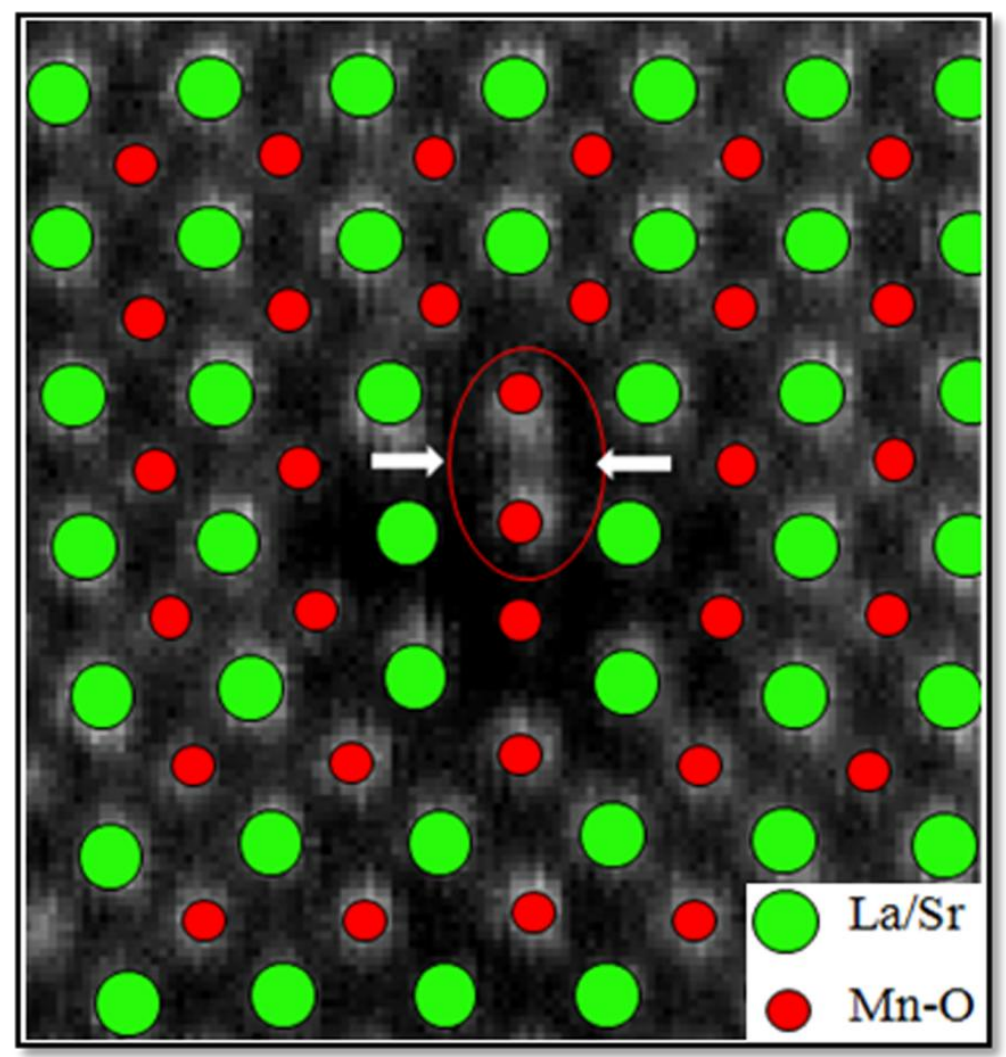

Figure. 5 Enlarged HAADF-STEM image around the edge dislocation core and the proposed structure model. The red circle indicates two Mn columns at the dislocation core and white arrows indicate two empty columns. Oxygen columns were not imaged here because of their low scattering factor.

\section{Conclusions}

In summary, with HAADF-STEM and atomic resolution STEM-EELS, we have investigated $\mathbf{a}<100>$ edge dislocation cores in $\mathrm{LSMO}$ film prepared on $\mathrm{LaAlO}_{3}$ substrate. The arrangements of $\mathrm{La}$ and $\mathrm{Mn}$ columns at dislocation cores are determined by HAADF-STEM image combined with STEM-EELS. The results of atomic resolution STEM-EELS spectra imaging indicate that the dislocation cores are nonstoichiometric with decreased $\mathrm{La} / \mathrm{Mn}$ and $\mathrm{O} / \mathrm{Mn}$ ratios and existence of oxygen 
vacancies. The dislocation cores are terminated with Mn columns. Two Mn columns at the dislocation cores occupy La-sites in La-sublattice and thus form antisite defects at the dislocation cores. This special dislocation core configuration might result from the accommodation of high-level strain at the dislocation cores.

\section{Acknowledgements}

The authors would like to thank the financial support from the National Science Foundation of China (grant numbers 51521091, 51371177 and 51390473). Bo Wu is acknowledged for the help with the microscope experiments.

\section{Reference}

Arredondo, M., Ramasse, Q. M., Weyland, M., Mahjoub, R., Vrejoiu, I., Hesse, D., Browning, N. D., Alexe, M., Munroe, P., Nagarajan, V., 2010. Direct evidence for cation non-stoichiometry and Cottrell atmospheres around dislocation cores in fuctional oxide interfaces. Adv. Mater. 22, 2430-2434.

Bosman, M., Keast, V. J., García-Muñoz, J. L., D’Alfonso, A. J., Findlay, S. D., Allen, L. J., 2007. Two-dimensional mapping of chemical information at atomic resolution. Phys. Rev. L. 99, 086102.

Chisholm, M. F., Pennycook, S. J., 2006. Direct imaging of dislocation core structures by Z-contrast STEM. Philo. Mag. 86, 29-31.

Cohen, R. E., 1992. Origin of ferroelectricity in perovskite oxides. Nature, 358, 136-138. 
Egerton, R. F., 2011. Electron Energy-Loss Spectroscopy in the Electron Microscope, third ed. Springer, US

Kim, M., Duscher, G., Browning, N. D., Sohlberg, K., Pantelides, S. T., Pennycook, S. J., 2001. Nonstoichiometry and the electrical activity of grain boundaries in $\mathrm{SrTiO}_{3}$. Phys. Rev. L. 86, 4056-4056.

Klie, R. F., Buban, J.P., Varela, M., Franceschetti, A., Jooss, C., Zhu, Y., Browning, N. D., Pantelides, S. T., Pennycook, S. J., 2005. Enhanced current transport at grain boundaries in high-Tc superconductors. Nature, 435, 475-478.

Kurata, H., Isojima, S., Kawai, M., Shimakawa, Y., Isoda, S., 2008. Local analysis of the edge dislocation core in $\mathrm{BaTiO}_{3}$ thin film by STEM-EELS. J. Micron. 236, $128-131$.

Leapman, R. D., Grunes, L. A., Fejes, P. L., 1982. Study of the L23 edges in the 3d transition metals and their oxides by electron-energy-loss spectroscopy with comparisons to theory. Phys. Rev. B. 26, 614-635.

Lee, J. H., Fang, L., Vlahos, E., Ke, X., Jung, Y. W., Kourkoutis, L. F., Kim, J., Ryan, P. J., Heeg, T., Roeckerath, M., Goian, V., Bernhagen, M., Uecker, R., Hammel, P. C., Rabe, K. M., Kamba, S., Schuber, J., Freeland, J. W., Muller, D. A., Fennie, C. J., Schiffer, P., Gopalan, V., Johnston-Halperin, E., Schlom, D. G., 2010. A strong ferroelectric ferromagnet created by means of spin-lattice coupling. Nature, 466, 954-958.

Millis, A. J., Shraiman, B. I., Mueller, R., 1996. Dynamic Jahn-Teller effect and colossal magnetoresistance in $\mathrm{La}_{1-\mathrm{x}} \mathrm{Sr}_{\mathrm{x}} \mathrm{MnO}_{3}$. Phys. Rev. Lett. 77, 175-178. 
Muller, D. A., 2009. Structure and bonding at the atomic scale by scanning transmission electron microscopy. Nat. Mater. 8, 263-270.

Pennycook, S. J., Boatner, C. A., 1988. Chemically Sensitive Structure-Imaging with a Scanning-Transmission Electron-Microscope, Nature, 336, 565-567.

Perovic, D. D., Rossouw, C. J., Howie, A., 1993. Imaging elastic strains in high-angle annular dark field scanning transmission electron microscopy. Unltramicroscopy, 52, 353-359.

Qin, Y. L., Jia, C. L., Urban, K., Hao, J. H., Xi, X. X., 2002. Dislocations in $\mathrm{SrTiO}_{3}$ thin films grown on LaAlO3 substrates. J. Mater. Res. 17, 3117-3126.

Reyren, N., Thiel, S., Caviglia, A. D., Kourkoutis, L. F., Hammerl, G., Richter, C., Schneider, C. W., Kopp, T., Rüetschi, A.-S., Jaccard, D., Gabay, M., Muller, D. A., Triscone, J. M., Mannhart, J., 2007. Superconducting interfaces between insulating oxides. Science, $317,1196-1199$.

Rez, P., 1982. Cross-sections for energy loss spectrometry. Ultramicroscropy, 9, 283-287.

Schmid, H. K., Mader, W., 2009. Oxidation states of Mn and Fe in various compound oxide systems. Micron, 37, 426-432.

Sugiyama, I., Shibata, N., Wang, Z., Kobayashi, S., Yamamoto, T., Ikuhara, Y., 2013. Ferromagnetic dislocations in antiferromagnetic NiO. Nat. Nanotechnol. 8, 266-270. Suzuki, T., Nishi, Y., Fujimoto, M., 1999. Analysis of misfit relaxation in heteroepitaxial $\mathrm{BaTiO}_{3}$ thin films. Philo. Mag. A. 79, 2461-2483.

Varela, M., Findlay, S. D., Lupini, A. R., Christen, H. M., Borisevich, A. Y., Dellby, 
N., Krivanek, O. L., Nellist, P. D., Oxley, M. P., Allen, L. J., Pennycook, S. J., 2004. Spectroscopic imaging of single atoms within a bulk solid. Phys. Rev. L. 92, 095502. Varela, M., Oxley, M. P., Luo, W., Tao, J., Watanabe, M., Lupini, A. R., Pantelides, S. T., Pennycook, S. J., 2009. Atomic-resolution imaging of oxidation states in manganites. Phys. Rev. B. 79, 085117.

Zhang, Z., Sigle, W., Kurts, W., 2004. HRTEM and EELS study of screw dislocation cores in $\mathrm{SrTiO}_{3}$. Phys. Rev. B. 69, 144103.

Zhang, Z., Sigle, W., Kurtz, W., Ruhle, M., 2002. Electronic and atomic structure of a dissociated dislocation in $\mathrm{SrTiO}_{3}$. Phys. Rev. B. 66, 214112. 


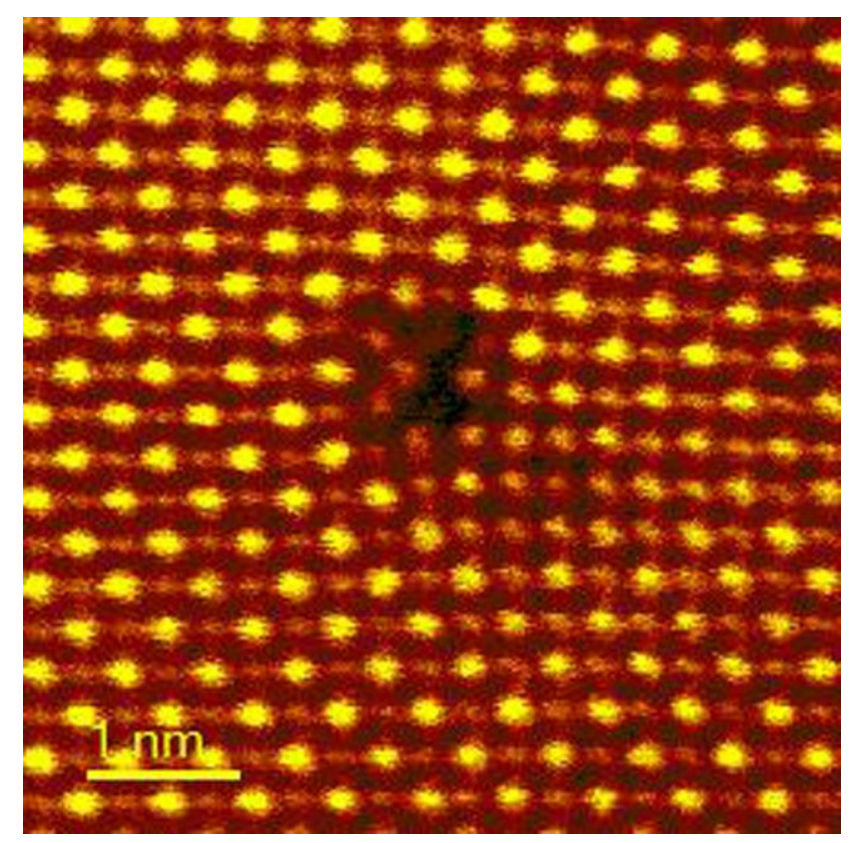

